Meta

Journal des traducteurs

Translators' Journal

\title{
La traduction technique en France
}

\section{Daniel Moskowitz}

Volume 28, numéro 1, mars 1983

La traduction dans le monde

Translation around the World

URI : https://id.erudit.org/iderudit/003673ar

DOI : https://doi.org/10.7202/003673ar

Aller au sommaire du numéro

Éditeur(s)

Les Presses de l'Université de Montréal

ISSN

0026-0452 (imprimé)

1492-1421 (numérique)

Découvrir la revue

Citer cet article

Moskowitz, D. (1983). La traduction technique en France. Meta, 28(1), 90-92.

https://doi.org/10.7202/003673ar

Ce document est protégé par la loi sur le droit d'auteur. L'utilisation des services d'Érudit (y compris la reproduction) est assujettie à sa politique d'utilisation que vous pouvez consulter en ligne.

https://apropos.erudit.org/fr/usagers/politique-dutilisation/
Cet article est diffusé et préservé par Érudit.

Érudit est un consortium interuniversitaire sans but lucratif composé de l’Université de Montréal, l'Université Laval et l'Université du Québec à Montréal. Il a pour mission la promotion et la valorisation de la recherche. https://www.erudit.org/fr/ 


\section{LA TRADUCTION TECHNIQUE EN FRANCE}

Daniel Moskowitz

Qu'est-ce que la traduction technique? On distingue traditionnellement «traduction littéraire» et «traduction technique». Il est assez facile de cerner la notion de traduction littéraire, mais beaucoup plus difficile de définir la traduction technique. Selon les utilisateurs, ce terme désigne :

1) toutes les traductions non littéraires

2) toutes les traductions qui ne sont pas destinées à la publication en librairie

3) les traductions industrielles ou technologiques

4) les traductions scientifiques et technologiques.

Il serait souhaitable que les milieux professionnels s'habituent à parler de traduction opératoire ou de traduction pragmatique (J. Delisle) par opposition à la traduction littéraire; cette solution mettrait fin aux multiples subdivisions, d'un emploi plus ou moins fréquent : traduction juridique, médicale, administrative, financière, économique, etc.

En France on compte quatre formes principales d'exercice du métier de traducteur : $l^{e}$ entreprises de traduction; $2^{e}$ traducteurs indépendants; $3^{e}$ traducteurs salariés; $4^{e}$ traducteurs et réviseurs d'organisations internationales (UNESCO, OCDE, etc.).

La Société Française des traducteurs (SFT) compte actuellement $850 \mathrm{mem}$ bres environ. Une estimation donne : $50 \%$ de traducteurs indépendants; $25 \%$ de traducteurs salariés; $25 \%$ de traducteurs littéraires.

Cette estimation est très grossière car les traducteurs n'aiment pas préciser la nature de leurs activités. Ils craignent toujours de perdre un travail, d'où un «flou artistique» dans les réponses à tous les questionnaires relatifs à la profession.

La profession de traducteur n'est en outre pas réglementée en France. De très nombreuses personnes font des traductions sans que cela constitue leur activité principale : retraités, professeurs de langue, étudiants, etc. Des sondages permettent de penser que la SFT ne regroupe ainsi que 20 à $25 \%$ des personnes exerçant une activité de traducteur à temps partiel ou complet.

Le marché de la traduction en France a beaucoup souffert de la réduction des activités du secteur tertiaire depuis la crise pétrolière, et surtout depuis 1979-80. Le poste de traductions est éminement compressible et le marché du travail est ainsi très sensible à l'évolution de la situation économique. La crise économique est toutefois loin d'expliquer à elle seule la situation actuelle de crise de la traduction. Parmi les nombreux facteurs d'influence, on peut citer : 
- les publications en anglais. De très nombreux scientifiques français publient leurs travaux en anglais, même dans des revues acceptant les textes en français. Cette attitude est dictée par un souci de renommée internationale, une plus grande diffusion des travaux, le désir de faire carrière, etc. Le gouvernement et divers organismes, dont notamment l'Académie nationale de médecine, tentent de redresser la situation dans ce domaine, mais il faut bien reconnaître que les résultats ne sont guère encourageants pour l'instant.

- application laxiste des textes réglementaires. La loi $\mathrm{n}^{\circ}$ 75-1349 du 31 décembre 1975 relative à l'emploi de la langue française stipule dans son article $1 \mathrm{er}$ :

Dans la désignation, l'offre, la présentation, la publicité écrite ou parler, le mode d'emploi ou d'utilisation, l'étendue et les conditions de garantie d'un bien ou d'un service, ainsi que les factures et quittances, l'emploi de la langue française est obligatoire. Le recours à tout terme étranger ou à toute expression étrangère est prohibé lorsqu'il existe une expression ou un terme approuvés dans les conditions prévues par le décret $\mathrm{n}^{\circ} 72-19$ du 7 janvier 1972 relatif à l'enrichissement de la langue française[...]

Les mêmes règles s'appliquent à toutes informations ou présentations de programmes de radiodiffusion et de télévision, sauf lorsqu'elles sont destinées expressément à un public étranger.

Il est évident que l'application stricte de cette loi ouvrirait d'importants débouchés à la traduction. Il suffit pour s'en convaincre de lire les innombrables modes d'emploi, notices d'utilisation, instructions de service, etc. fournis avec les biens, matériels ou équipements vendus en France. Tous ces documents sont soit en anglais, soit traduits dans le pays exportateur par des personnes n'ayant aucune qualification professionnelle. Ce problème fait actuellement l'objet d'une étude au niveau ministériel.

- recours à des traducteurs non professionnels. Une enquête effectuée auprès des scientifiques d'une université parisienne en 1980 a montré que les $3 / 4$ des auteurs traduisent eux-mêmes leurs travaux en anglais, bien que plus de $80 \%$ des personnes interrogées estiment que la traduction est une opération difficile ou très difficile! La raison le plus fréquemment avancée pour justifier ce comportement est l'absence de crédit et de traducteurs qualifiés. Ce problème est lié à la promotion du français langue scientifique et à la diffusion de la culture scientifique et technique, sur lesquelles nous reviendrons ci-après. Beaucoup trop d'entreprises, grandes ou petites, ont par ailleurs la déplorable habitude de confier leurs travaux de traduction «au moins disant» afin de dépenser le moins possible. Cette façon de procéder contribue à l'actuel marasme de la traduction et se traduit presque toujours par une qualité très médiocre des traductions.

L'École supérieure d'interprètes et de traducteurs (ESIT) de l'Université de Paris III est une école professionnelle de niveau universitaire. La grande majorité de ses enseignants sont des traducteurs professionnels. L'ESIT dispose ainsi avec les enseignants et les anciens élèves diplômés d'une information rapide et précise sur l'évolution du marché. L'état de ce dernier a des répercussions sensibles au niveau de l'ESIT. 
1) Contraitement à ce qui se passait naguère, les jeunes traducteurs diplômés ont du mal à trouver leur premier emploi. Le délai moyen de première embauche est ainsi passé en quelques années de 2-3 semaines à quelques mois. Il semble que la combinaison linguistique du traducteur intervienne moins que par le passé et que les exigences de qualité deviennent par contre de plus en plus sévères. Par raison d'économie, les employeurs exigent de plus en plus souvent que le traducteur débutant soit immédiatement opérationnel. Il est parfaitement admis qu'un jeune ingénieur par exemple a besoin de plusieurs mois pour se former aux techniques maison. On demande par contre au jeune traducteur de connaître dès le départ l'ensemble des techniques et toute la terminologie spécifique, ce qui est évidemment impossible et rend la découverte du premier emploi de plus en plus difficile.

2) La crise économique affectant tous les secteurs d'activité, la traduction apparaît de plus en plus - et malgré les difficultés précitées - comme un secteur d'avenir. Le nombre de candidats à l'examen d'admission de l'ESIT en 1982 a augmenté de plus d'un tiers par rapport à celui de 1981 .

3) Exigence de qualité croissante. Toujours par raison d'économie, le employeurs souhaitent n'avoir qu'un nombre limité de traducteurs, mais de très haute qualité. La polyvalence est de plus en plus souvent exigée, ce qui ne manque pas de réagir sur les programmes d'enseignement.

Le gouvernement français a récemment manifesté à plusieurs reprises son intérêt pour la défense de la langue française d'une part, la recherche et le développement technologique d'autre part. La loi $\mathrm{n}^{\circ} 82-610$ du 15 juillet 1982 d'orientation et de programmation pour la recherche et le développement technologique de la France comporte en annexe un rapport sur la programmation et l'orientation de l'effort national de recherche et de développement technologique. Dans la section «Promotion du français langue scientifique et diffusion de la culture scientifique et technique» ce rapport précise: «Cet effort implique notamment une grande politique de la traduction, une activité soutenue de création terminologique...»

Divers services ministériels déploient actuellement une grande activité en vue de la création d'une banque de terminologie française, du développement de services de néologie et de terminologie, du développement de la traduction assistée par ordinateur. Plusieurs commissions d'experts se réunissent fréquemment pour fixer les objectifs, déterminer les procédures à mettre en œuvre et estimer les moyens nécessaires.

M. Pierre Mauroy, Premier ministre, vient en outre de demander pour la fin février 1983 une étude attentive des problèmes soulevés par «le développement des professions de traducteur et d'interprète, vecteurs de la transmission de la pensée, de la recherche et de la technologie dans le monde».

Il est probable que toutes les actions envisagées ne pourront pas être menées simultanément, mais il est permis d'espérer que tous les efforts entrepris aboutiront rapidement à une amélioration de la situation de la traduction technique en France : meilleure connaissance des besoins, assistance à la formation de haute niveau professionnel, prévision systématique de la traduction dans les organigrammes et les projets de recherche, création de services de traduction à la disposition des scientifiques, etc. 